\title{
mRNA-seq reveals skeletal muscle atrophy in response to handling stress in a marine teleost, the red cusk-eel (Genypterus chilensis)
}

\author{
Jorge E. Aedo ${ }^{1}$, Jonathan Maldonado ${ }^{5}$, Víctor Aballai ${ }^{1}$, Juan M. Estrada ${ }^{4}$, Macarena Bastias-Molina ${ }^{6}$, \\ Claudio Meneses ${ }^{6}$, Cristian Gallardo-Escarate ${ }^{2,3}$, Herman Silva ${ }^{5}$, Alfredo Molina ${ }^{1,3,4}$ and Juan A. Valdés ${ }^{1,3,4^{*}}$
}

\begin{abstract}
Background: Fish reared under intensive conditions are repeatedly exposed to stress, which negatively impacts growth. Although most fish follow a conserved pattern of stress response, with increased concentrations of cortisol, each species presents specificities in the cell response and stress tolerance. Therefore, culturing new species requires a detailed knowledge of these specific responses. The red cusk-eel (Genypterus chilensis) is a new economically important marine species for the Chilean aquaculture industry. However, there is no information on the stress- and cortisol-induced mechanisms that decrease skeletal muscle growth in this teleost.

Results: Using Illumina RNA-seq technology, skeletal muscle sequence reads for G. chilensis were generated under control and handling stress conditions. Reads were mapped onto a reference transcriptome, resulting in the in silico identification of 785 up-regulated and 167 down-regulated transcripts. Gene ontology enrichment analysis revealed a significant upregulation of catabolic genes associated with skeletal muscle atrophy. These results were validated by RT-qPCR analysis for ten candidates genes involved in ubiquitin-mediated proteolysis, autophagy and skeletal muscle growth. Additionally, using a primary culture of fish skeletal muscle cells, the effect of cortisol was evaluated in relation to red cusk-eel skeletal muscle atrophy.

Conclusions: The present data demonstrated that handling stress promotes skeletal muscle atrophy in the marine teleost $\mathrm{G}$. chilensis through the expression of components of the ubiquitin-proteasome and autophagy-lysosome systems. Furthermore, cortisol was a powerful inductor of skeletal muscle atrophy in fish myotubes. This study is an important step towards understanding the atrophy system in non-model teleost species and provides novel insights on the cellular and molecular mechanisms that control skeletal muscle growth in early vertebrates.
\end{abstract}

Keywords: Genypterus chilensis, Red cusk-eel, mRNA-seq, Handling stress, Skeletal muscle atrophy, Cortisol

\section{Background}

Over the past decades, the aquaculture industry has significantly advanced in terms of technology and rearing methods due to the growing demand of fish for human consumption [1]. While many different species are cultured worldwide, the Chilean finfish industry is highly

\footnotetext{
* Correspondence: jvaldes@unab.cl

'Laboratorio de Biotecnología Molecular, Facultad de Ciencias Biológicas,

Universidad Andrés Bello, Santiago, Chile

${ }^{3}$ Interdisciplinary Center for Aquaculture Research (INCAR), P.O. Box 160-C, Concepción, Chile

Full list of author information is available at the end of the article
}

concentrated in salmonid farming $[2,3]$. However, there is a recent local and global trend towards diversifying breeding species to maintain the sustainability of the aquaculture industry $[4,5]$. In Chile, one such cultivated marine species is the red cusk-eel (Genypterus chilensis, Guichenot, 1881), a teleost of the Ophidiidae family [6]. This fish is highly valued in national and international markets due to exceptional flesh quality and high nutritional value $[7,8]$. Nevertheless, the culturing of this species is seriously hindered by scarce biological knowledge and, primarily, limited information on the negative 
effects of stress associated with intensive farming in marine species [9].

The stress response in fish occurs when a stimulus is perceived as a threat. This response increases cortisol secretion, as mediated through the hypothalamic-pituitaryinterrenal axis [10]. If the stress is chronic, affected individuals could have permanently increased circulating cortisol levels, a situation that involves short-term metabolic changes and a long-term response associated with reduced growth [11]. An important tissue for growth regulation is skeletal muscle, which is fundamental for an organism's metabolism and physiology [12, 13]. In mammals, it is well documented that under stressful pathological conditions increased cortisol levels are associated to skeletal muscle atrophy results from increased protein breakdown and decreased protein synthesis [14], however relatively little is known about this condition in teleost skeletal muscle. The two major route that increases overall rates of protein degradation during muscle atrophy are the ubiquitin-proteasome and the autophagy-lysosome systems [15]. The stimulation by cortisol of these two protein degradation pathways are mediated through the increased expression of several atrogenes, such as Atrogin-1 [16], Foxo [17] and MURF-1 [18], as well as other intracellular mediators related to autophagy such as KLF15 [19], and REDD1 [20]. Although a few studies have gone further in the understanding of skeletal muscle response in relation to different stressors in teleosts by using PCR arrays [21, 22], microarray [23-26], and RNA-seq approaches [27-30], there are no studies focused to understand the relevance of cortisol in the skeletal muscle response to stress.

It was only recently that the first annotated transcriptome of G. chilensis was published through the use of Illumina technology, thus providing valuable transcriptomic information for this species and for members of the Ophidiidae family [31]. The aim of the present study was to characterize the effect of the stress caused by handling on the skeletal muscle of G. chilensis, an economically important marine fish in the Chilean aquaculture industry. For this, Illumina reads obtained from control and stressed fish were mapped onto the reference transcriptome of the red cusk-eel to identify differentially expressed transcripts (DETs). Gene ontology enrichment analysis revealed a significant up-regulation of catabolic genes associated with skeletal muscle atrophy. Using in vitro approximations, it was also determined that cortisol-mediated genomic actions are a powerful inductor of skeletal muscle atrophy in fish. In the short term, these results will aid in modifying rearing protocols, the aim of which is to improve animal welfare. Importantly, these results also contribute towards the overall understanding of the atrophy system in a non-model teleost, providing novel insights on the cellular and molecular mechanisms that control skeletal muscle growth in early vertebrates.

\section{Results}

Physiological and transcriptomic responses of red cusk-eel skeletal muscle to handling stress

Juvenile red cusk-eels were stressed daily with a netting and chasing protocol, and samples were obtained after five days of this procedure. Plasma cortisol and glucose levels significantly increased (5.3-fold and 1.7-fold, respectively) as compared to the control fish, revealing the magnitude of the stress induced by handling. However, no significant differences in plasma lactate levels were observed (Fig. 1). Two separate cDNA libraries were constructed from the skeletal muscle of pooled juvenile red cusk-eels from control and stressed conditions. To examine sequencing variations, cDNA library replicates of each

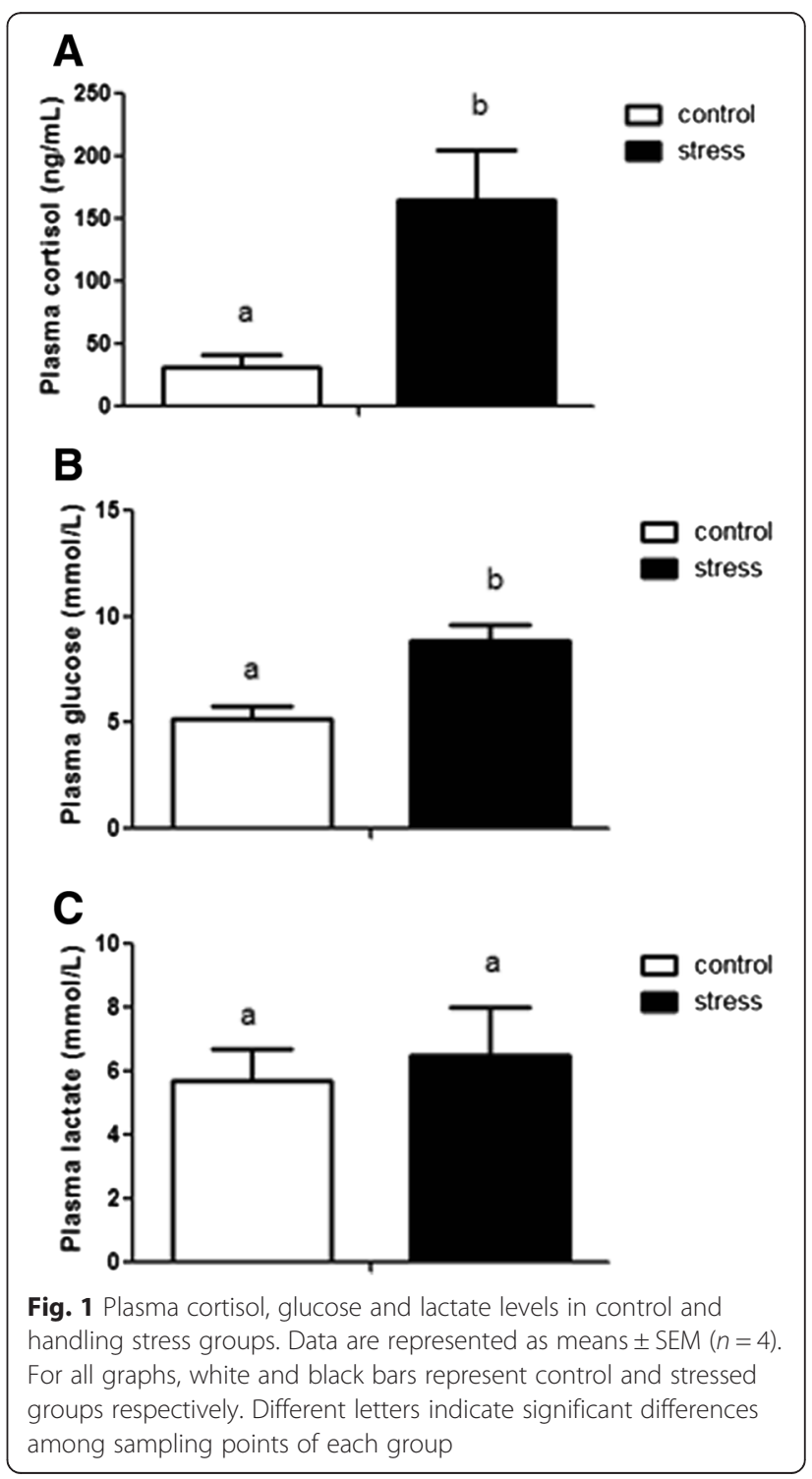


condition were constructed. In total, Illumina MiSeq sequencing generated 23,731,898 paired-end reads (Table 1). Raw data were deposited in the NCBI Sequence Read Archive under Accession Number [GenBank SRS614525: SRR2060847, SRR2063782, SRR2064146, SRR2064148]. After trimming adapters, low quality base pairs, and short reads, the two sequence sets were reduced to 22,992,184 high-quality reads (Table 1). These reads were analyzed with the CLC Genomic Workbench software v.7.0.3 using the previously reported reference transcriptome for G. chilensis [31], resulting in $\sim 98.8 \%$ of the reads mapped. The expression level of each transcript was represented as RPKM, with 785 transcripts up-regulated under stressed conditions and 167 transcripts down-regulated under stressed conditions. A complete list of the differentially expressed transcripts is included in Additional file 1: Table S1.

\section{Gene ontology (GO) enrichment, KEGG pathway analysis and $\mathrm{QPCR}$ validation}

The DAVID gene functional classification tool was used to identify groups of transcripts sharing common GO terms. The transcripts up-regulated under stressed conditions were significantly enriched in biological processes, such as modification-dependent macromolecule catabolic process (GO:0043632), modification-dependent protein catabolic process (GO:0019941), and cellular protein catabolic process (GO:0044257), among others (Table 2). The down-regulated transcripts under stressed conditions were significantly enriched in biological processes including striated muscle contraction (GO:0006941), muscle organ development (GO:0007517), and muscle contraction (GO:0006936), among others (Table 2). The GO terms for up-regulated transcripts were significantly enriched in molecular functions, such as ubiquitin-protein ligase activity (GO:0004842), acid-amino acid ligase activity (GO:0016881), and small conjugating protein ligase activity (GO:0019787), among others (Additional file 2: Table S2). The GO terms for down-regulated transcripts were significantly enriched in molecular functions, such as cytoskeletal protein binding (GO:0008092), structural constituent of muscle (GO:0008307), actin binding (GO:0003779), among others (Additional file 2: Table S2). The enrichment of up-regulated transcripts in cellular component distribution were in membrane-enclosed lumen (GO:0031974), organelle lumen (GO:0043233), and intracellular organelle lumen (GO:0070013), among others (Additional file 3: Table S3). The enrichment of downregulated transcripts in cellular component distribution were in contractile fiber part (GO:0044449), sarcomere (GO:0030017), and contractile fiber (GO:0043292), among others (Additional file 3: Table S3). Finally, pathway analysis through KEGG revealed up-regulated transcripts to be enriched in ubiquitin-mediated proteolysis, regulation of autophagy, and Proteasome (Additional file 4: Table S4). The down-regulated transcripts were enriched in KEGG pathways such as muscle contraction, hypertrophic cardiomyopathy, and dilated cardiomyopathy (Additional file 4: Table S4).

PathVisio 3.0 was used to visualize and integrate the transcriptomic data obtained in KEGG pathway analysis, related to ubiquitin-mediated proteolysis, regulation of autophagy, and muscle contraction (Fig. 2). 21 transcripts associated to the ubiquitin-mediated proteolysis were up-regulated such as components of the E1- ubiquitin activating enzyme (uba1), E2-ubiquitin conjugating enzyme (ube2b, ube2g1,ube2q,ube2r), E3-ubiquitin ligase (ube3a, ube3c, nedd4, herc2, ube 4b, mdm2, pirh2, $f b x 032$ ), as well as components of the $26 \mathrm{~S}$ proteasome (psmd1, psmd4, psmd5, psmd7, psmd8, psmd11, psmd14, psme1). Similarly, 10 transcripts associated to the regulation of autopaghy were up-regulated such as components of mTOR signaling (ampk, redd1, ulk1, pi3kciii) and components that control autophagosome formation (atg9, atg5, atg16l, atg4, atg7, lc3i). Oppositely, 17 transcripts associated to muscle contraction were down-regulated such as components of the Z-disk (actn2, actn3), myosin heavy chain (myh2, myh3, myh4, myh7, myh10), myosin binding (mybpc1, mybpc3), tropomyosin (tpm1), troponin (tnnt2, tnnt3, tnni1, tnnc1), actin chain (acta1), and myosin light chain (myl3, myl4). Additionally, transcription factors involved in skeletal muscle growth (myod1, myod2) and transcription factors involved in skeletal muscle atrophy (smad2, foxo1) were incorporated.

Eight up-regulated transcripts related to skeletal muscle atrophy (foxo1, ddit4, psmd1, smad2, fbx032, eif4ebp3, atg5, and atg16l1) and two down-regulated transcripts related to skeletal muscle growth (myod1 and

Table 1 Summary of sequencing and mapping results

\begin{tabular}{lllllll}
\hline Condition & Number of reads & Average length & $\begin{array}{l}\text { Number of reads } \\
\text { after trimming }\end{array}$ & $\begin{array}{l}\text { Average length } \\
\text { after trimming }\end{array}$ & $\begin{array}{l}\text { Percent of } \\
\text { mapped reads }\end{array}$ & $\begin{array}{l}\text { Percent of mapped reads } \\
\text { with functional anotation }\end{array}$ \\
\hline Control & $5,369,110$ & 157.2 & $5,261,728$ & 153.1 & 98.9 & 43.4 \\
Control (replicate) & $6,475,266$ & 152.4 & $6,268,257$ & 151.9 & 99.1 & 43.5 \\
Handling stress & $5,037,796$ & 170.2 & $4,488,662$ & 157.5 & 98.7 & 43.3 \\
Handling stress (replicate) & $6,849,726$ & 165.5 & $6,575,737$ & 156.2 & 98.5 & 43.2 \\
Total/average & $23,731,898$ & 161.3 & $22,992,184$ & 154.7 & 98.8 & 43.4 \\
\hline
\end{tabular}


Table 2 Enriched biological processes of up-regulated and down-regulated transcripts in response to handling stress

\begin{tabular}{|c|c|c|c|}
\hline GO ID & GO term & $p$-value & Fold enrichment \\
\hline & \multicolumn{3}{|c|}{ Enrichment of up regulated transcripts in skeletal muscle under stress } \\
\hline GO:0043632 & Modification-dependent macromolecule catabolic process & $2.3 \mathrm{E}-06$ & 3.8 \\
\hline GO:0019941 & Modification-dependent protein catabolic process & $2.3 \mathrm{E}-06$ & 3.8 \\
\hline GO:0044257 & Cellular protein catabolic process & $3.6 \mathrm{E}-06$ & 2.7 \\
\hline GO:0008104 & Protein localization & $6.8 \mathrm{E}-06$ & 2.8 \\
\hline GO:0015031 & Protein transport & $7.4 \mathrm{E}-06$ & 2.8 \\
\hline GO:0045184 & Establishment of protein localization & $7.4 \mathrm{E}-06$ & 3.4 \\
\hline GO:0051603 & Proteolysis involved in cellular protein catabolic process & $9.1 \mathrm{E}-06$ & 3.4 \\
\hline GO:0044265 & Cellular macromolecule catabolic process & $1.2 \mathrm{E}-05$ & 3.2 \\
\hline GO:0046907 & Intracellullar transport & $1.4 \mathrm{E}-05$ & 3.3 \\
\hline GO:0030163 & Protein catabolic process & $3.0 \mathrm{E}-05$ & 3.1 \\
\hline GO:0009057 & Macromolecule catabolic process & $8.4 \mathrm{E}-05$ & 2.8 \\
\hline GO:0006396 & RNA processing & $3.3 \mathrm{E}-04$ & 2.8 \\
\hline \multirow[t]{2}{*}{ GO:0006511 } & Ubiquitin-dependent protein catabolic process & $4.4 \mathrm{E}-03$ & 3.0 \\
\hline & \multicolumn{3}{|c|}{ Enrichment of down regulated transcripts in skeletal muscle under stress } \\
\hline GO:0006941 & Striated muscle contraction & $1.9 \mathrm{E}-06$ & 4.3 \\
\hline GO:0007517 & Muscle organ development & $4.3 \mathrm{E}-06$ & 4.2 \\
\hline GO:0006936 & Muscle contraction & $5.5 \mathrm{E}-06$ & 5,7 \\
\hline GO:0003012 & Muscle system process & $2.3 \mathrm{E}-05$ & 3.5 \\
\hline GO:0014706 & Striated muscle tissue development & $3.1 \mathrm{E}-05$ & 4.9 \\
\hline GO:0060537 & Muscle tissue development & $5.8 \mathrm{E}-05$ & 6.7 \\
\hline GO:0048738 & Cardiac muscle tissue development & $6.6 \mathrm{E}-05$ & 6.7 \\
\hline GO:0043462 & Regulation of ATPase activity & $9.5 \mathrm{E}-05$ & 3.5 \\
\hline GO:0060048 & Cardiac muscle contraction & $2.5 \mathrm{E}-03$ & 4.2 \\
\hline GO:0006937 & Regulation of muscle contraction & $6.5 \mathrm{E}-03$ & 3.1 \\
\hline
\end{tabular}

myod2) were selected for RT-qPCR analysis so as to validate the results of RNA-seq analysis. The transcript expression fold-changes measured by these two methods (Fig. 3) were highly correlated, with a significant $\mathrm{R}^{2}$ value of $0.85\left(p\right.$-value $\left.=1 \mathrm{E}^{-4}\right)$. Taken together, these results indicate that stress induces red cusk-eel skeletal muscle atrophy and suggest that cortisol modulates the expression of components from the ubiquitin-proteasome and autophagy systems.

\section{Cellular and molecular response of red cusk-eel myotubes to cortisol}

To determine if cortisol was the main modulator of skeletal muscle atrophy in red cusk-eel, the mRNA expression of foxo1, ddit4, psmd1, smad2, fbx032, eif4ebp3, atg5, atg16l, myod1 and myod2 were monitored in myotube lysates 12,24 , and $36 \mathrm{~h}$ following cortisol treatment $(250 \mathrm{ng} / \mathrm{mL}$ ) (Fig. 4a-i). At $12 \mathrm{~h}$ post-treatment, maximum increases in ddit4 and eif4ebp3 mRNA expressions were observed. At $24 \mathrm{~h}$ post-treatment, there was a maximum increase in foxo1 and smad2 mRNA expressions, which was followed by maximum increases of psmd1, fbx032, atg5, and atg16l mRNA expressions at $36 \mathrm{~h}$ posttreatment with cortisol. Pretreatment of skeletal myotubes with the glucocorticoid receptor antagonist RU486 (1 $\mu \mathrm{M})$ significantly inhibited the cortisolinduced up-regulation of foxo1, ddit4, psmd1, smad2, $f b x 032$, eif4ebp3, atg5 and atg16l. No changes in gene expression were observed for myod 1 or myod 2 following cortisol treatment (data not shown). To determine whether cortisol treatment effectively induced atrophy in fish myotubes, protein ubiquitination levels were recorded $36 \mathrm{~h}$ post-treatment. Increased ubiquitination occurred after treatment, and this increase was inhibited by pretreatment with RU486 (Fig. 4j). Additionally, cortisol treatment triggered a significant decrease in myotube diameters, in association with muscular atrophy, at five days post-treatment (Fig. 4k). These results indicate that cortisol, through their genomic action, is a powerful inductor of skeletal muscle atrophy in fish myotubes. 


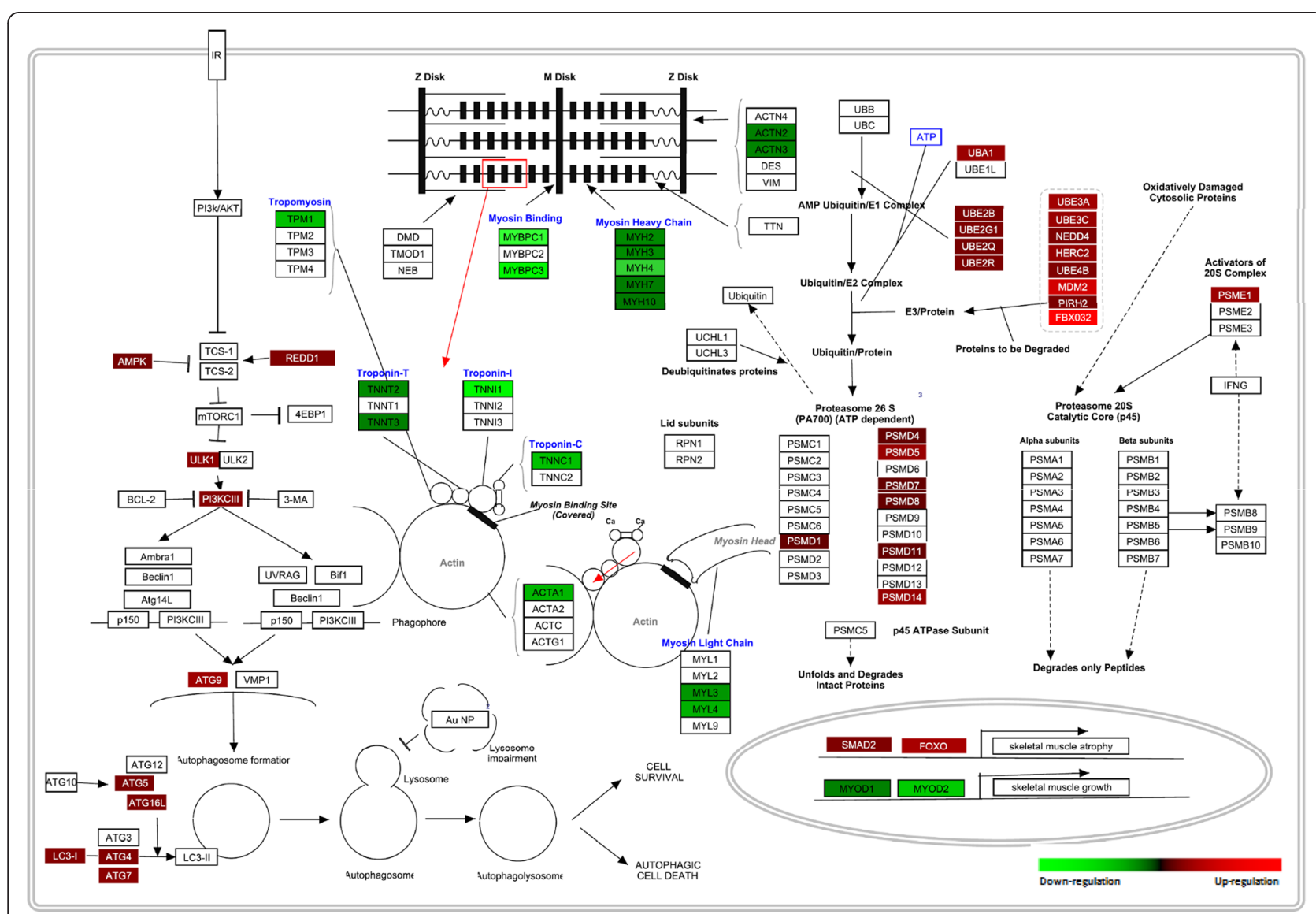

Fig. 2 Comprehensive illustration of transcription changes in skeletal muscle of red cusk-eel under handling stress using modified KEGG pathway maps using Pathvisio v3. The figure depicts ubiquitin-proteasome. autophagy-lysosome and striated muscle contraction genes (indicated as rectangles)

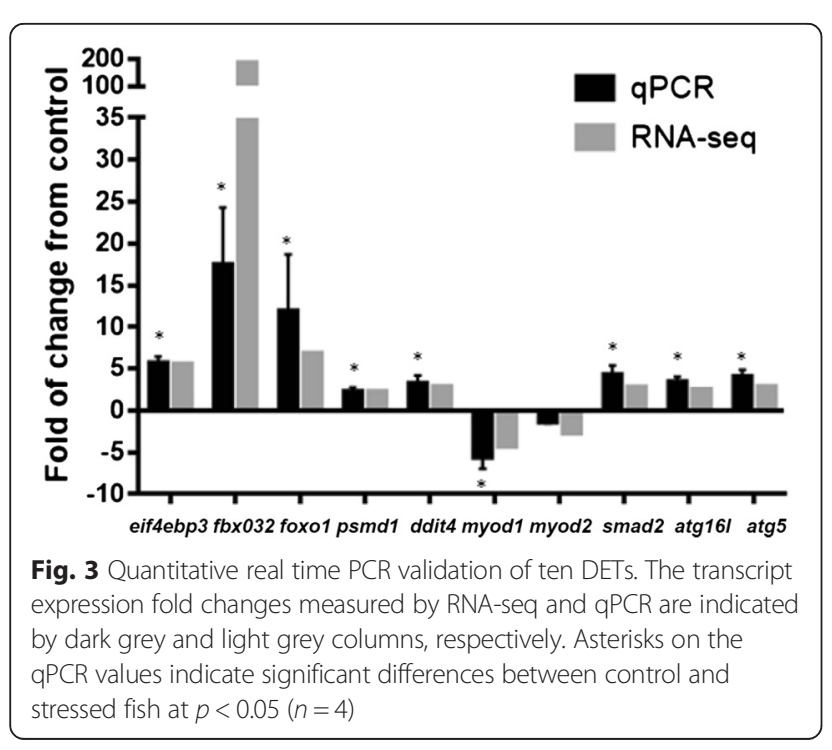

\section{Discussion}

Genypterus chilensis is an economically important marine species for Chilean aquaculture industry. The first reference transcriptome for this species was previously sequenced and annotated through Illumina HiSeq paired-end sequencing [31]. In this study, RNA-seq analysis was used to determine the effects of handling stress on the skeletal muscle of the red cusk-eel, revealing 952 DETs associated with an up-regulation of catabolic biological processes or a down-regulation of skeletal muscle contraction biological processes. The results obtained by this in silico approach were validated by a RT-qPCR analysis of genes representative of the most relevant identified processes, as well as by an in vitro approach that revealed interesting details regarding the regulation dynamics and mechanisms of cortisol-mediated skeletal muscle atrophy in fish. In general terms, the present study found the primary stress response associated to increased levels cortisol and glucose in plasma was very similar to other fish species, such as the rainbow trout (Oncorhynchus mykiss) [32] or Senegalese sole (Solea senegalensis) [33]. 


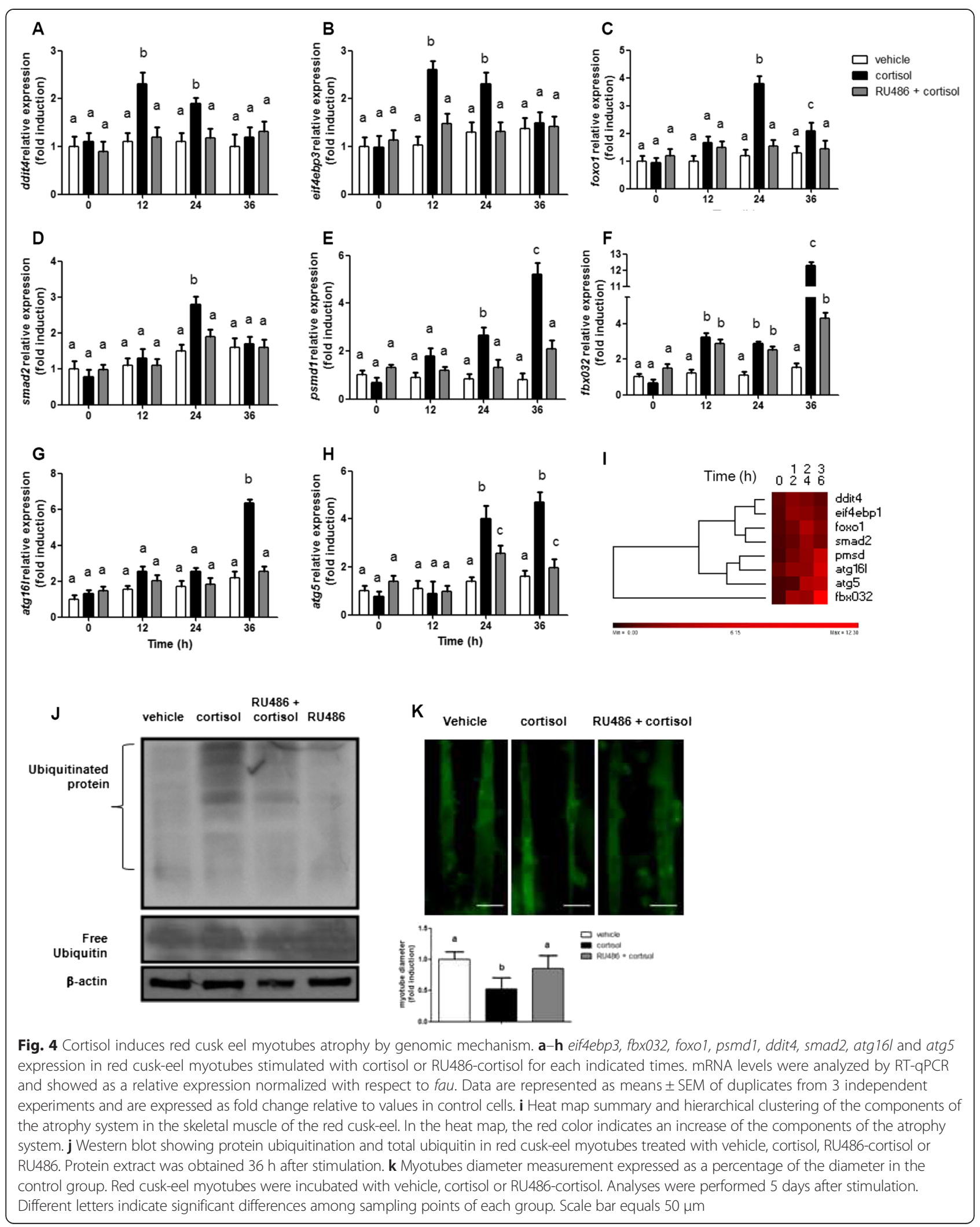


In teleosts, cortisol is the primary glucocorticoid and regulator of the physiological response to stress [34]. Cortisol binds and activates specific corticoid receptors in target tissues. In fish there are two types of corticoid steroid receptors: mineralocorticoid receptors (MR) and glucocorticoid receptors (GR) [35]. Most fish possess one MR and two GR isoforms, GR1 and GR2 [36]. Once inside the cell, cortisol binds to cytoplasmic GRs to induce conformational changes that cause the receptor to dissociate from respective chaperone molecules. The hormone-receptor complex then translocates to the nucleus where it dimerises and binds to the glucocorticoid response elements of target genes [37]. Depending on the co-factors recruited, this leads to the transactivation or transrepression of these genes. This mode of cortisol action is referred to as the classical or genomic pathway [37]. Cortisol-mediated stress is a key controller in aerobic and anaerobic metabolism [38], increasing gluconeogenesis, and inhibiting glycogen synthesis [39]. An increased metabolic rate contributes to reduced growth. Cortisol may also control growth by acting through elevated plasma glucose levels to reduce appetite and food ingestion [40]. Due to this, elevated cortisol levels could be inferred through reduced food conversion efficiency, and growth [40].

For years it was assumed that the low growth rates experienced by fish under stressful conditions were due to metabolic changes impacting anabolic processes [41]. However, the present work is the first to evidence that stress mediated by cortisol induces the expression of components associated with skeletal muscle catabolism. The transcripts up-regulated under stressed conditions were associated with catabolic pathways such as ubiquitin-mediated proteolysis and autophagy, while down-regulated transcripts were associated with muscle contraction or muscle hypertrophy pathways. Interestingly in humans, similar signatures have been observed in skeletal muscle atrophy induced by corticosteroids used to treat several pathological conditions or by cortisol released in response to stress conditions such as sepsis, cachexia, and starvation [42].

In mammals, in vitro models have been very useful in defining the glucocorticoid pathway by reducing complexity, as compared to an in vivo model $[43,44]$. Similarly, fish myotubes primary culture has been used as a valid model for understanding the signaling pathways involved in muscle development and growth in teleost, including for glucose transport [45], TOR signaling [46], IGF-1 signaling [47-49], Myostatin signaling [50, 51], GH signaling [52], proinflammatory cytokine signaling [53], PAMPs signaling [54], and cortisol signaling [55]. Similarly, in the present study using an in vitro model, it was determined that cortisol through the expression of components of the ubiquitin-proteasome and autophagy- lysosome systems was an inductor of skeletal muscle atrophy in fish,.

The maximum foxolexpression in myotubes occurred $24 \mathrm{~h}$ post-treatment with cortisol. The role of this transcription factor in skeletal muscle atrophy induced by glucocorticoids has been demonstrated in mammals through in vitro and in vivo approximations [56-58]. Moreover, a recent study demonstrated the presence of functional glucocorticoid response elements in the mammalian FoxO1 promoter [59]. In fish, the expression and activity of FoxO1 have been observed under nutritional stress in fine flounder (Paralichthys adspersus) [60]. Interestingly, IGF-1, an inductor of skeletal muscle hypertrophy, induced the phosphorylation of FoxO1 and FoxO4 in trout myotubes in association with decreased transcriptional activity [61]. The described FoxO1 target genes included atrogin-1 ( $b x \times 32)$, a protein involved in muscle proteolysis via the ubiquitin-proteasome system [17]. Atrogin-1 expression occurs in relation to skeletal muscle atrophy under fasting conditions in rainbow trout [62] and fine flounder [60]. Recently, it was determined that FOXO1/Atrogin-1 signaling pathway is involved in the skeletal muscle atrophy induced by LPS in rainbow trout myotubes [54].

Other important components of protein catabolism include the $26 \mathrm{~S}$ proteasome and autophagosome. The upregulation of the $26 \mathrm{~S}$ proteasome non-ATPase regulatory subunit 1 (psmd1) has been observed in atrophying fasttwitch muscles from rats treated with dexamethasone [63]; however, there are no reports on the differential expression of this gene in teleosts. A similar phenomenon has been reported for ATG5 and ATG16L1, where dexamethasone treatment in L6 myotubes induces the expression of several autophagy genes $24 \mathrm{~h}$ post-treatment, including of atg5 and atg16l1 [64]. In teleost species, ATG5 activity in zebrafish has been linked to neurogenesis and organogenesis [65]. Nevertheless, there are no reports that relate ATG5 gene expression with fish muscle atrophy.

Another gene with differential expression identified in this study was REDD1 (ddit4), a cytoplasmic protein with a crucial role in repressing protein synthesis mediated by TOR [66]. Additionally, the same signaling pathway showed an over expression of 4EBP-1 (eif4ebp3). This protein represses protein synthesis by directly interacting with the eukaryotic translation initiation factor $4 \mathrm{E}$ [66]. In both cases, the present study detected maximum expression $12 \mathrm{~h}$ post-treatment with cortisol, suggesting that the inhibition of protein synthesis precedes atrogene expression. In mammals, acute dexamethasone treatment induces REDD1 mRNA expression in rat skeletal muscle in vivo and in L6 myoblasts, as well as downregulating mTOR signaling through the activation of 4EBP-1 [67]. In zebrafish, REDD1 regulates dorsoventral 
patterning through the negative modulation of $\mathrm{Wnt} / \beta$ catenin activity [68].

Among the genes down-regulated under handling stress, the present study found proteins associated with contractile functions and development, including specific muscle transcription factors such as MyoD1 and MyoD2. Both proteins belong to the myogenic regulatory factors (MRFs) protein family and are involved in the regulation of teleost myogenesis [69]. While both transcripts had decreased expression in RNA-seq analysis, only myod 1 showed a significant decrease in its expression in RT-qPCR analysis. Moreover, changes in expression were not detected for myod 1 or myod 2 in fish myotubes treated with cortisol. This result differs from that observed in mammals, where treatment of the $\mathrm{C} 2 \mathrm{C} 12$ myoblast with dexamethasone reduces myod expression [70]. This observation can be explained by the differences between mammalian and teleost myogenesis [71].

\section{Conclusions}

The present work used RNA-seq analysis to determine the effects of handling stress on the skeletal muscle transcriptome of G. chilensis, an important marine fish for the Chilean aquaculture industry. Handling stress induced physiological changes, associated with increases in circulating levels of cortisol, and major changes in global skeletal muscle gene expression. Under stressed conditions, 785 transcripts were up-regulated in association with catabolic signaling pathway such as the ubiquitin-proteasome and autophagy-lysosome systems. Conversely, 167 transcripts were down-regulated under stressed conditions in association with biological processes such as muscle contraction and muscle system processes. The transcriptional expression of components from the ubiquitin-proteasome and autophagy-lysosome pathways were analyzed by RTqPCR and all were significantly increased under handling stress. To further analyze the relevance of cortisol in teleost skeletal muscle atrophy, an in vitro approximation was performed, providing details about the dynamics and mechanisms by which cortisol induces skeletal muscle atrophy in teleosts. There was a coordinated expression of genes related to the suppression of protein synthesis, protein ubiquitination, and autophagy. All of these were modulated by the genomic actions of cortisol. This is the first study to indicate that stress and cortisol are powerful inductors of skeletal muscle atrophy in a teleost, in addition to providing valuable information for monitoring the culturing and growth of marine fish species under intensive rearing conditions.

\section{Methods}

Fish sampling and stress conditions

Juvenile red cusk-eels (Genypterus chilensis) with an average weight of $900 \pm 50 \mathrm{~g}$ and length of $55 \pm 5 \mathrm{~cm}$ were collected from the Centro de Investigación Mar-

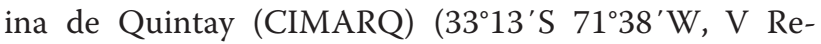
gion, Valparaíso, Chile). Fish were maintained under natural temperature and light:dark photoperiod conditions $\left(13{ }^{\circ} \mathrm{C} \pm 1{ }^{\circ} \mathrm{C}\right.$ and $\mathrm{L}: \mathrm{D}$ 12:12) for the spring season. The specimens were randomly distributed between a control and stressed group, placed in two separate $90 \mathrm{~L}$ tanks, and acclimated for 2 weeks before the experiment. The stressed group was then subjected to a standardized handling stress protocol consisting of netting and chasing the fish for 5 min daily for 5 days [32]. Six hours after the final handling stimulation, stressed and control fish were quickly netted, and blood samples were taken via caudal puncture using heparinized tubes ( $n=4$ per group). Plasma was collected by centrifugation at $5000 \times g$ for $10 \mathrm{~min}$ and stored at $-80^{\circ} \mathrm{C}$ until analysis. Following blood sampling, fish were sacrificed through an overdose of anesthetic (3aminobenzoic acid ethyl ester, $300 \mathrm{mg} / \mathrm{L}$ ), and white muscle was collected, immediately frozen in liquid nitrogen, and stored at $-80{ }^{\circ} \mathrm{C}$ until analysis. Fish were reared and sampled according to protocols approved by the Bioethical Committee of Andrés Bello University.

\section{Cortisol, glucose, and lactate measurements}

Plasma cortisol levels were measured using the Enzyme Immunoassay Kit (Cayman, MI, USA) following the manufacturer's recommendations. Glucose and lactate plasma levels were measured using Colorimetric Assay Kits (Abcam, Cambridge, UK) following the manufacturer's recommendations.

\section{Skeletal muscle RNA extraction and transcriptome sequencing}

Total RNA was extracted using the RNeasy Mini Kit (Qiagen, TX, USA) following the manufacturer's instructions. RNA was quantified through spectrophotometry using NanoDrop technology with the Epoch Multi-Volume Spectrophotometer System (BioTek, VT, USA). Total RNA isolated from skeletal muscle was treated with DNase I to remove genomic DNA. RNA concentration was mesuared by Qubit $^{\circ}$ 2.0 Fluorometer (Life Technology, Carlsbad, CA, USA) and RNA integrity was determined using Fragment Analyzer $^{\mathrm{rm}}$ Automated CE System (Analytical Advanced Technologies, Ames, IA, USA). Library construction of $1 \mu \mathrm{g}$ total RNA for each sample was made using Illumina ${ }^{\circ}$ TruSeq $^{\circ}$ RNA Sample prep kit (Illumina ${ }^{\circ}$, USA), according to protocol indications, with mRNA fragmentation time of 2 min at $94{ }^{\circ} \mathrm{C}$. After PCR amplification step, mRNA libraries sizes were verified on AATI Fragment Analyzer ${ }^{\mathrm{Tm}}$, and quantified by qPCR-based quantification using Kapa Library Quantification kit (\#KK4824). Libraries were diluted and prepared to a final concentration of $12,5 \mathrm{pM}$ for loading on Illumina ${ }^{\circ}$ MiSeq desktop sequencer, according to the 
MiSeq System User guide. cDNA library replicates of each condition were constructed and sequenced.

\section{Data processing, differentially expressed transcripts, and $\mathrm{GO}$ enrichment analysis}

Raw sequencing reads were trimmed by removing Illumina adapter sequences and low quality bases. Sequences shorter than 50 base pairs were also discarded. To analyze in silico gene expression levels, the CLC Genomics Workbench v.7.0.3 software (http://www.clcbio.com/genomics/) was used with the G. chilensis reference transcriptome [31]. RNA-seq analysis was carried out for sequence reads obtained from control and stressed conditions using the following default parameters: two mismatches, minimum fraction length of 0.9 , minimum fraction similarity of 0.8 , and a maximum of 10 hits per read. Gene expressions were based on reads per kilobase of exon model per million mapped reads (RPKM) values [72]. The up- and down-regulated genes were analyzed for gene ontology (GO) and Kyoto Encyclopedia of Genes and Genomes (KEGG) pathways through the DAVID database [73] and categorized based on GO terms for biological processes, molecular functions, and cellular components, as well as by genes up- or down-regulated in association with KEGG pathways. To establish a relationship between red cusk-eel DETs and DAVID background, a tBLASTx search was conducted against Danio rerio Ensembl proteins for significant matches with the red cusk-eel transcriptome. $D a$ nio rerio Ensembl Gene IDs were obtained from the corresponding Ensembl protein entries. DAVID analysis was performed with custom IDs set as the 'Background'. Standard settings for gene count (2) and ease (0.1) were used. The cut-off $p$-value for biological processes was $1 \mathrm{E}^{-6}$ and cut-off $p$-value for molecular functions and cellular components was $1 \mathrm{E}^{-3}$.

\section{Cell cultures and cortisol treatments}

Primary myoblasts were isolated from juvenile red cuskeels. Dorsal white muscle was obtained under sterile conditions and placed in an F10 medium containing $9 \mathrm{mM} \mathrm{NaHCO}, 20 \mathrm{mM}$ HEPES, $15 \%$ horse serum, 100 $\mathrm{U} / \mathrm{ml}$ penicillin, and $10 \mathrm{mg} / \mathrm{ml}$ streptomycin at $\mathrm{pH}$ 7.4. After mechanical dissociation of the muscle, the tissue was digested with a $0.1 \%$ collagenase and $0.1 \%$ trypsin solution in an F10 medium for $4 \mathrm{~h}$ at $15^{\circ} \mathrm{C}$. The suspension was centrifuged at $300 \times g$ for $5 \mathrm{~min}$ at $10^{\circ} \mathrm{C}$, and the resulting pellet was resuspended in an F10 medium. The cellular suspension was filtered through $40 \mu \mathrm{m}$ nylon filters. The cells were centrifuged at $1000 \times g$ for $10 \mathrm{~min}$ at $15{ }^{\circ} \mathrm{C}$, resuspended in $3 \mathrm{ml}$ of cold PBS, and layered on top of $4 \mathrm{ml}$ of Ficoll-Paque gradient (GE Healthcare) in a $15 \mathrm{ml}$ tube. Samples were then centrifuged at $1400 \times g$ for $30 \mathrm{~min}$ at $15^{\circ} \mathrm{C}$. Following this, the cell layer was extracted with a pipette and washed with
$10 \mathrm{ml}$ of F10 medium. Cells were seeded at a density of $2 \times 10^{6}$ per $\mathrm{mL}$ in plates previously treated with poly-Llysine and laminin. Cells were incubated for seven days at $15{ }^{\circ} \mathrm{C}$ under atmospheric air in a proliferating medium containing the F10 medium, $9 \mathrm{mM} \mathrm{NaHCO}, 20 \mathrm{mM}$ HEPES, $10 \%$ fetal bovine serum, $100 \mathrm{U} / \mathrm{ml}$ penicillin, and $10 \mathrm{mg} / \mathrm{ml}$ streptomycin. Then, myoblasts were cultivated an additional seven days in a differentiating medium composed by the F10 medium, $9 \mathrm{mM} \mathrm{NaHCO}$, $20 \mathrm{mM}$ HEPES, $100 \mathrm{U} / \mathrm{ml}$ penicillin, and $10 \mathrm{mg} / \mathrm{ml}$ streptomycin to obtain differentiated myotubes. On the fourteenth day, myotubes were treated with a medium containing either ethanol (vehicle control), cortisol $(250 \mathrm{ng} / \mathrm{mL})$, or RU486 $(1 \mu \mathrm{M})$. After 12,24 , and $36 \mathrm{~h}$, the medium was removed, and cells were harvested for total RNA isolation or protein extraction. All treatments were performed with $n=4$, in two independent experiments.

\section{Real-time qPCR}

Total RNA was extracted from skeletal muscle tissue and skeletal myotubes using the RNeasy Mini Kit (Qiagen, TX, USA) following the manufacturer's recommendations. RNA was quantified by spectrophotometry using NanoDrop technology with the Epoch Multi-Volume Spectrophotometer System (BioTek, VT, USA). Only RNAs with an A260/280 ratio between 1.9 and 2.1 were used for cDNA synthesis. Residual genomic DNA was removed using the genomic DNA wipeout buffer included in the Quantitect Reverse Transcription Kit (Qiagen, TX, USA). Subsequently, $1 \mu \mathrm{g}$ of RNA was reverse transcribed into cDNA for 30 min at $42{ }^{\circ} \mathrm{C}$ using the manufacturer's recommendations. qPCR analysis was performed with the Stratagene MX3000P qPCR System (Stratagene, CA, USA). Each qPCR reaction mixture contained $7.5 \mu \mathrm{l}$ of $2 \times$ Brilliant II SYBR Master Mix (Stratagene, CA, USA), $6 \mu \mathrm{l}$ of cDNA (diluted 40 -fold), and $250 \mathrm{nM}$ of each primer. For gene expression normalization, the reference gene 40S ribosomal protein S30 (fau) was used [74]. The primers used in this study are listed in Additional file 5: Table S5. Control reactions included a no template control and a control without reverse transcriptase. The QGene program was used for analysis of gene expression [75].

\section{Western blot analysis}

After treatment, cells were solubilized at $4{ }^{\circ} \mathrm{C}$ in $30 \mu \mathrm{L}$ of lysis buffer containing $50 \mathrm{mM}$ Tris- $\mathrm{HCl} \mathrm{pH} \mathrm{7.4,}$ $150 \mathrm{mM} \mathrm{NaCl}, 1 \mathrm{mM}$ EDTA, $1 \% \mathrm{NP}-40,5 \mathrm{mM}$ $\mathrm{Na}_{3} \mathrm{VO}_{4}, 20 \mathrm{mM} \mathrm{NaF}, 10 \mathrm{mM}$ sodium pyrophosphate, and a protease inhibitor cocktail (Calbiochem). Proteins extracts were resolved by $10 \%$ SDS-PAGE, transferred to polyvinylidene difluoride membranes (Millipore, MA, USA), and blocked for $1 \mathrm{~h}$ at room temperature in Tris-buffered saline, $0.1 \%$ Tween 20 , and $5 \%$ fatfree milk. Incubations with primary antibodies (1:1000) 
were performed at $4{ }^{\circ} \mathrm{C}$ overnight. After incubation for $1 \mathrm{~h}$ with horseradish peroxidase (HRP)-conjugated secondary antibodies (1:2000), membranes were developed through enhanced chemiluminescence (Amersham Biosciences, Amersham, UK). The films were scanned, and the ImageJ program was employed for densitometric analysis of the bands [76]. Antibodies against ubiquitin (cat. no. 2880), $\beta$ actin (cat. no. 4967), and HRP-conjugated secondary antirabbit and anti-mouse were obtained from Cell Signaling Technology (MA, USA).

\section{Myotube diameter measurements}

Myotubes were loaded with $5.4 \mathrm{mM}$ Calcein AM for $45 \mathrm{~min}$ at $15{ }^{\circ} \mathrm{C}$ in an $\mathrm{F} 10$ medium. Myotubes were transferred to the recording chamber and mounted on an inverted fluorescence microscope. Fluorescence was detected using excitation at $488 \mathrm{~nm}$ and emission at $540 \mathrm{~nm}$, and images were collected. The diameters were measured for a total of 50 myotubes from random fields using the Image J program [76]. Myotubes were measured at three points along their length, and results were expressed as a percentage of the diameter in the control group $(n=8)$ in two independent experiments.

\section{Pathway visualization and Heat map summary of atrophy data}

The construction of pathway maps of ubiquitin-proteasome, autophagy-lysosome and striated muscle contraction and visualization of differentially expressed gene were performed using PathVisio v3 [77]. The appropriate gene database was selected, and the expression dataset was created after importing the saved input file using the Import Expression Dataset tab.

The relationships among relative expression profiles, a heat map summary and hierarchical clustering analysis of gene expression was performed using Permutmatrix [78]. Clustering and seriation were based on Pearson's correlation coefficient of $\mathrm{z}$-score normalized relative transcript abundance values. McQuitty's method as hierarchical clustering was used.

\section{Statistical analysis}

Data are expressed as the mean \pm SE. Differences in means between groups were determined using one-way ANOVA followed by Bonferroni's post-test. Data were accepted as significant at a value of $P<0.05$. Correlations between RNA-seq and RT-qPCR data were assessed through multiple linear regression, which obtained the coefficient of determination $\left(\mathrm{R}^{2}\right)$ and $p$-value. All statistical analyses were performed using the program GraphPad Prism v.5.00 (GraphPad Software, CA, USA).

\section{Additional files}

Additional file 1: Table S1. Complete list of up-regulated and downregulated transcripts in response to handling stress. (XLSX 66 kb)

Additional file 2: Table S2. Enriched Molecular functions of upregulated and down-regulated transcripts in response to handling stress. (XLSX $10 \mathrm{~kb}$ )

Additional file 3: Table S3. Enriched cellular component of upregulated and down-regulated transcripts in response to handling stress. (XLSX $10 \mathrm{~kb}$ )

Additional file 4: Table S4. Enriched KEGG pathways of up-regulated and down-regulated transcripts in response to handling stress. (XLSX $10 \mathrm{~kb}$ )

Additional file 5: Table S5. Primer sequences for qPCR assay, amplicon size and PCR. (XLSX $10 \mathrm{~kb}$ )

\section{Abbreviations}

FPKM: Fragments per kilobase of exon model per million mapped reads; GO: Gene ontology; DET: Differentially expressed transcript.

\section{Competing interests}

The authors declare that they have no competing interests.

\section{Authors' contributions}

AM and JV conceived and designed the study. JV wrote the manuscript. CM and MB performed the Illumina Sequencing. JA and JM analyzed the RNA Seq data and performed statistical analyses. JA and VA performed the RT-qPCR and in vitro experiments. CG, HS and JME contributed analysis, text and comments to the paper. All authors read and approved the final manuscript.

\section{Acknowledgements}

This study was funded by the National Commission for Scientific and Technological Research (CONICYT), FONDAP projects 15110027 and 15090007.

\section{Author details}

${ }^{1}$ Laboratorio de Biotecnología Molecular, Facultad de Ciencias Biológicas, Universidad Andrés Bello, Santiago, Chile. 'Laboratory of Biotechnology and Aquatic Genomics, Universidad de Concepción, Concepción, Chile. ${ }^{3}$ Interdisciplinary Center for Aquaculture Research (INCAR), P.O. Box 160-C, Concepción, Chile. ${ }^{4}$ Centro de Investigación Marina Quintay (CIMARQ), Universidad Andrés Bello, Quintay, Chile. ${ }^{5}$ Departamento de Producción Agrícola, Laboratorio de Genómica Funcional \& Bioinformática, Universidad de Chile, Facultad de Ciencias Agronómicas, Av. Santa Rosa 11315, La Pintana, 8820808 Santiago, Chile. ${ }^{6}$ Centro de Biotecnología Vegetal, Facultad Ciencias Biológicas, Universidad Andrés Bello, Santiago, Chile.

Received: 16 June 2015 Accepted: 19 November 2015 Published online: 01 December 2015

\section{References}

1. FAO: Fisheries and Aquaculture Department. The State of World Fisheries and Aquaculture 2012. Rome: FAO; 2012.

2. Ibieta P, Tapia V, Venegas $C$, Hausdorf M, Takle H. Chilean Salmon Farming on the Horizon of Sustainability: Review of the Development of a Highly Intensive Production, the ISA Crisis and Implemented Actions to Reconstruct a More Sustainable Aquaculture Industry. In: Sladonja B, editor. Aquaculture and the Environment - A Shared Destiny. Croatia: InTech; 2011.

3. lizuka M, Katz J. Globalisation, Sustainability and the Role of Institutions: The Case of the Chilean Salmon Industry: Globalisation, Sustainability and the Role of Institutions. Tijdschr Econ Soc Geogr. 2015;106:140-53.

4. François NRL, Carter C. Finfish Aquaculture Diversification. New York: CABl; 2010.

5. Chirivella J, Mañanós EL, Muñoz JL, Soler E. Diversification of farmed species. España: Ministerio de Medio Ambiente y Medio Rural y Marino; 2011.

6. Nielsen JG, editor. Ophidiiform Fishes of the World (order Ophidiiformes):: An Annotated and Illustrated Catalogue of Pearlfishes, Cusk-eels, Brotulas, and Other Ophidiiform Fishes Known to Date. Rome: Food and Agriculture Organization of the United Nations; 1999 [FAO Species Catalogue, v. 18]. 
7. Vega R, Pradenas M, Estrada JM, Ramirez D, Valdebenito I, Mardones A, et al. Evaluacion y comparacion de la eficiencia de dos sistemas de incubacion de huevos de Genypterus chilensis (Guichenot, 1848). Latin Am J Aquat Res. 2012:40:187-200.

8. Chong J, Gonzalez P. Reproductive cycle and maturity mean size of the red cusk eel, Genypterus chilensis (Guichenot, 1881) in the coast off Talcahuano, Chile. Rev Biol Mar Oceanogr. 1881;2009(44):257-62.

9. Vijayan MM, Moon TW. The stress response and the plasma disappearance of corticosteroid and glucose in a marine teleost, the sea raven. Can J Zool. 1994;72:379-86.

10. Wendelaar Bonga SE. The stress response in fish. Physiol Rev. 1997;77:591-625.

11. Ellis T, Yildiz HY, López-Olmeda J, Spedicato MT, Tort L, Øverli $\varnothing$, et al. Cortisol and finfish welfare. Fish Physiol Biochem. 2012;38:163-88.

12. Johnston IA. Physiology of muscle in hatchery raised fish. Comp Biochem Physiol B Biochem. 1982;73:105-24.

13. Weatherley $\mathrm{AH}$, Gill HS. Dynamics of increase in muscle fibers in fishes in relation to size and growth. Experientia. 1985;41:353-4.

14. Braun TP, Marks DL. The regulation of muscle mass by endogenous glucocorticoids. Front Physiol. 2015;6:12.

15. Sandri M. Protein breakdown in muscle wasting: Role of autophagylysosome and ubiquitin-proteasome. Int J Biochem Cell Biol. 2013:45:2121-9.

16. Gomes MD, Lecker SH, Jagoe RT, Navon A, Goldberg AL. Atrogin-1, a muscle-specific F-box protein highly expressed during muscle atrophy Proc Natl Acad Sci U S A. 2001;98:14440-5.

17. Sandri M, Sandri C, Gilbert A, Skurk C, Calabria E, Picard A, et al. Foxo Transcription Factors Induce the Atrophy-Related Ubiquitin Ligase Atrogin-1 and Cause Skeletal Muscle Atrophy. Cell. 2004;117:399-412.

18. Waddell DS, Baehr LM, van den Brandt J, Johnsen SA, Reichardt HM, Furlow JD, et al. The glucocorticoid receptor and FOXO1 synergistically activate the skeletal muscle atrophy-associated MuRF1 gene. Am J Physiol Endocrinol Metab. 2008:295:E785-97.

19. Shimizu N, Yoshikawa N, Ito N, Maruyama T, Suzuki Y, Takeda S, et al. Crosstalk between glucocorticoid receptor and nutritional sensor mTOR in skeletal muscle. Cell Metab. 2011;13:170-82.

20. Britto FA, Begue G, Rossano B, Docquier A, Vernus B, Sar C, et al. REDD1 deletion prevents dexamethasone-induced skeletal muscle atrophy. Am J Physiol Endocrinol Metab. 2014;307:E983-93.

21. Bermejo-Nogales A, Calduch-Giner JA, Pérez-Sánchez J. Unraveling the molecular signatures of oxidative phosphorylation to cope with the nutritionally changing metabolic capabilities of liver and muscle tissues in farmed fish. PLoS ONE. 2015;10:e0122889.

22. Calduch-Giner JA, Echasseriau Y, Crespo D, Baron D, Planas JV, Prunet $P$, et al. Transcriptional Assessment by Microarray Analysis and Large-Scale Metaanalysis of the Metabolic Capacity of Cardiac and Skeletal Muscle Tissues to Cope With Reduced Nutrient Availability in Gilthead Sea Bream (Sparus aurata L.). Mar Biotechnol. 2014;16:423-35.

23. Salem M, Kenney PB, Rexroad CE, Yao J. Microarray gene expression analysis in atrophying rainbow trout muscle: a unique nonmammalian muscle degradation model. Physiol Genomics. 2006;28:33-45.

24. Johansen KA, Sealey WM, Overturf K. The effects of chronic immune stimulation on muscle growth in rainbow trout. Comp Biochem Physiol B Biochem Mol Biol. 2006;144:520-31.

25. Magnoni LJ, Roher N, Crespo D, Krasnov A, Planas JV. In Vivo Molecular Responses of Fast and Slow Muscle Fibers to Lipopolysaccharide in a Teleost Fish, the Rainbow Trout (Oncorhynchus mykiss). Biology (Basel). 2015;4:67-87.

26. Nesan D, Vijayan MM. The transcriptomics of glucocorticoid receptor signaling in developing zebrafish. PLOS ONE. 2013;8:e80726.

27. Garcia de la Serrana D, Devlin RH, Johnston IA. RNAseq analysis of fast skeletal muscle in restriction-fed transgenic coho salmon (Oncorhynchus kisutch): an experimental model uncoupling the growth hormone and nutritional signals regulating growth. BMC Genomics. 2015;16:564

28. Sun S, Xuan F, Fu H, Zhu J, Ge X, Gu Z. Transciptomic and histological analysis of hepatopancreas, muscle and gill tissues of oriental river prawn (Macrobrachium nipponense) in response to chronic hypoxia. BMC Genomics. 2015;16:491.

29. Salem M, Manor ML, Aussanasuwannakul A, Kenney PB, Weber GM, Yao J. Effect of sexual maturation on muscle gene expression of rainbow trout: RNA-Seq approach. Physiol Rep. 2013;1:e00120.

30. Palstra AP, Beltran S, Burgerhout E, Brittijn SA, Magnoni LJ, Henkel CV, et al. Deep RNA Sequencing of the Skeletal Muscle Transcriptome in Swimming Fish. PLOS ONE. 2013:8:e53171.
31. Aedo JE, Maldonado J, Estrada JM, Fuentes EN, Silva H, Gallardo-Escarate C, et al. Sequencing and de novo assembly of the red cusk-eel (Genypterus chilensis) transcriptome. Mar Genomics. 2014;18:105-7.

32. Krasnov A, Koskinen H, Pehkonen P, Rexroad CE, Afanasyev S, Mölsä H. Gene expression in the brain and kidney of rainbow trout in response to handling stress. BMC Genomics. 2005;6:3.

33. Cordeiro OD, Silva TS, Alves RN, Costas B, Wulff T, Richard N, et al. Changes in liver proteome expression of Senegalese sole (Solea senegalensis) in response to repeated handling stress. Mar Biotechnol. 2012;14:714-29.

34. Pankhurst NW. The endocrinology of stress in fish: an environmental perspective. Gen Comp Endocrinol 2011;170:265-275.

35. Greenwood AK, Butler PC, White RB, DeMarco U, Pearce D, Fernald RD. Multiple corticosteroid receptors in a teleost fish: distinct sequences, expression patterns, and transcriptional activities. Endocrinology. 2003;144:4226-36.

36. Baker ME, Funder JW, Kattoula SR. Evolution of hormone selectivity in glucocorticoid and mineralocorticoid receptors. J Steroid Biochem Mol Biol. 2013;137:57-70.

37. Aluru N, Vijayan MM. Stress transcriptomics in fish: A role for genomic cortisol signaling. Gen Comp Endocrinol. 2009;164:142-50.

38. De Boeck G, Alsop D, Wood C. Cortisol effects on aerobic and anaerobic metabolism, nitrogen excretion, and whole-body composition in juvenile rainbow trout. Physiol Biochem Zool. 2001;74:858-68.

39. Milligan $\mathrm{CL}$. A regulatory role for cortisol in muscle glycogen metabolism in rainbow trout Oncorhynchus mykiss Walbaum. J Exp Biol. 2003;206(Pt 18):3167-73.

40. Bernier NJ, Bedard N, Peter RE. Effects of cortisol on food intake, growth, and forebrain neuropeptide $Y$ and corticotropin-releasing factor gene expression in goldfish. Gen Comp Endocrinol. 2004;135:230-40.

41. Oliva-Teles A. Nutrition and health of aquaculture fish: Nutrition and health of aquaculture fish. J Fish Dis. 2012:35:83-108.

42. Calura E, Cagnin S, Raffaello A, Laveder P, Lanfranchi G, Romualdi C. Meta-analysis of expression signatures of muscle atrophy: gene interaction networks in early and late stages. BMC Genomics. 2008;9:630.

43. Croxtall JD, van Hal PTW, Choudhury Q, Gilroy DW, Flower RJ. Different glucocorticoids vary in their genomic and non-genomic mechanism of action in A549 cells. Br J Pharmacol. 2002;135:511-9.

44. Sanden S, Tripmacher R, Weltrich R, Rohde W, Hiepe F, Burmester GR, et al. Glucocorticoid dose dependent downregulation of glucocorticoid receptors in patients with rheumatic diseases. J Rheumatol. 2000;27:1265-70.

45. Díaz M, Vraskou Y, Gutiérrez J, Planas JV. Expression of rainbow trout glucose transporters GLUT1 and GLUT4 during in vitro muscle cell differentiation and regulation by insulin and IGF-I. Am J Physiol Regul Integr Comp Physiol. 2009;296:R794-800

46. Seiliez I, Gabillard J-C, Skiba-Cassy S, Garcia-Serrana D, Gutiérrez J, Kaushik S, et al. An in vivo and in vitro assessment of TOR signaling cascade in rainbow trout (Oncorhynchus mykiss). Am J Physiol Regul Integr Comp Physiol. 2008;295:R329-35.

47. Castillo J, Le Bail P-Y, Paboeuf G, Navarro I, Weil C, Fauconneau B, et al. IGF-I binding in primary culture of muscle cells of rainbow trout: changes during in vitro development. Am J Physiol Regul Integr Comp Physiol. 2002;283:R647-52.

48. Castillo J, Codina M, Martínez ML, Navarro I, Gutiérrez J. Metabolic and mitogenic effects of IGF-I and insulin on muscle cells of rainbow trout. Am J Physiol Regul Integr Comp Physiol. 2004;286:R935-41.

49. Castillo J, Ammendrup-Johnsen I, Codina M, Navarro I, Gutiérrez J. IGF-I and insulin receptor signal transduction in trout muscle cells. Am J Physiol Regul Integr Comp Physiol. 2006;290:R1683-90.

50. Garikipati DK, Rodgers BD. Myostatin stimulates myosatellite cell differentiation in a novel model system: evidence for gene subfunctionalization. Am J Physiol Regul Integr Comp Physiol. 2012;302:R1059-66.

51. Garikipati DK, Rodgers BD. Myostatin inhibits myosatellite cell proliferation and consequently activates differentiation: evidence for endocrine-regulated transcript processing. J Endocrinol. 2012;215:177-187.

52. Levesque HM, Shears MA, Fletcher GL, Moon TW. Myogenesis and muscle metabolism in juvenile Atlantic salmon (Salmo salar) made transgenic for growth hormone. J Exp Biol. 2008;211(Pt 1):128-37.

53. Pooley NJ, Tacchi L, Secombes CJ, Martin SA. Inflammatory responses in primary muscle cell cultures in Atlantic salmon (Salmo salar). BMC Genomics. 2013;14:747.

54. Aedo JE, Reyes AE, Avendaño-Herrera R, Molina A, Valdés JA. Bacterial lipopolysaccharide induces rainbow trout myotube atrophy via Akt/FoxO1/ Atrogin-1 signaling pathway. Acta Biochim Biophys Sin. 2015;47:932-7. 
55. Galt NJ, Froehlich JM, Remily EA, Romero SR, Biga PR. The effects of exogenous cortisol on myostatin transcription in rainbow trout, Oncorhynchus mykiss. Comp Biochem Physiol A Mol Integr Physiol. 2014;175:57-63.

56. Cho JE, Fournier M, Da X, Lewis MI. Time course expression of Foxo transcription factors in skeletal muscle following corticosteroid administration. J Appl Physiol. 2010;108:137-45.

57. Gilson H, Schakman O, Combaret L, Lause P, Grobet L, Attaix D, et al. Myostatin gene deletion prevents glucocorticoid-induced muscle atrophy. Endocrinology. 2007;148:452-60.

58. Sanchez AMJ, Candau RB, Bernardi H. FoxO transcription factors: their roles in the maintenance of skeletal muscle homeostasis. Cell Mol Life Sci. 2014;71:1657-71.

59. Qin W, Pan J, Qin Y, Lee DN, Bauman WA, Cardozo C. Identification of functional glucocorticoid response elements in the mouse FoxO1 promoter. Biochem Biophys Res Commun. 2014;450:979-83.

60. Fuentes EN, Ruiz P, Valdes JA, Molina A. Catabolic signaling pathways, atrogenes, and ubiquitinated proteins are regulated by the nutritional status in the muscle of the fine flounder. PLoS ONE. 2012;7:e44256.

61. Seiliez I, Sabin N, Gabillard J-C. FoxO1 is not a key transcription factor in the regulation of myostatin (mstn-1a and mstn-1b) gene expression in trout myotubes. Am J Physiol Regul Integr Comp Physiol. 2011;301:R97-104.

62. Cleveland BM, Evenhuis JP. Molecular characterization of atrogin-1/F-box protein-32 (FBXO32) and F-box protein-25 (FBXO25) in rainbow trout (Oncorhynchus mykiss): Expression across tissues in response to feed deprivation. Comp Biochem Physiol B Biochem Mol Biol. 2010;157:248-57.

63. Combaret L, Taillandier D, Dardevet D, Béchet D, Rallière C, Claustre A, et al. Glucocorticoids regulate mRNA levels for subunits of the $19 \mathrm{~S}$ regulatory complex of the $26 \mathrm{~S}$ proteasome in fast-twitch skeletal muscles. Biochem J. 2004:378(Pt 1):239-46.

64. Troncoso R, Paredes F, Parra V, Gatica D, Vásquez-Trincado C, Quiroga C, et al. Dexamethasone-induced autophagy mediates muscle atrophy through mitochondrial clearance. Cell Cycle. 2014;13:2281-95.

65. Hu Z, Zhang J, Zhang Q. Expression pattern and functions of autophagyrelated gene atg5 in zebrafish organogenesis. Autophagy. 2011;7:1514-27.

66. Schakman O, Kalista S, Barbé C, Loumaye A, Thissen JP. Glucocorticoidinduced skeletal muscle atrophy. Int J Biochem Cell Biol. 2013;45:2163-72.

67. Wang H, Kubica N, Ellisen LW, Jefferson LS, Kimball SR. Dexamethasone represses signaling through the mammalian target of rapamycin in muscle cells by enhancing expression of REDD1. J Biol Chem. 2006;281:39128-34.

68. Feng Q, Zou X, Lu L, Li Y, Liu Y, Zhou J, et al. The stress-response gene redd1 regulates dorsoventral patterning by antagonizing Wnt/ $\beta$-catenin activity in zebrafish. PLOS ONE. 2012;7:e52674.

69. Gabillard JC, Sabin N, Paboeuf G. In vitro characterization of proliferation and differentiation of trout satellite cells. Cell Tissue Res. 2010;342:471-7.

70. Te Pas MF, de Jong PR, Verburg FJ. Glucocorticoid inhibition of $\mathrm{C} 2 \mathrm{C} 12$ proliferation rate and differentiation capacity in relation to mRNA levels of the MRF gene family. Mol Biol Rep. 2000;27:87-98.

71. Rossi G, Messina G. Comparative myogenesis in teleosts and mammals. Cell Mol Life Sci. 2014;71:3081-99.

72. Mortazavi A, Williams BA, McCue K, Schaeffer L, Wold B. Mapping and quantifying mammalian transcriptomes by RNA-Seq. Nat Methods. 2008:5:621-8.

73. Huang DW, Sherman BT, Lempicki RA. Systematic and integrative analysis of large gene lists using DAVID bioinformatics resources. Nat Protoc. 2008;4:44-57.

74. Fuentes EN, Safian D, Valdés JA, Molina A. Isolation and selection of suitable reference genes for real-time PCR analyses in the skeletal muscle of the fine flounder in response to nutritional status: assessment and normalization of gene expression of growth-related genes. Fish Physiol Biochem. 2013;39:765-77.

75. Simon P. Q-Gene: processing quantitative real-time RT-PCR data. Bioinformatics. 2003;19:1439-40.

76. Schneider CA, Rasband WS, Eliceiri KW. NIH Image to ImageJ: 25 years of image analysis. Nat Methods. 2012;9:671-5

77. Van lersel MP, Kelder T, Pico AR, Hanspers K, Coort S, Conklin BR, et al. Presenting and exploring biological pathways with PathVisio. BMC Bioinformatics. 2008;9:399.

78. Caraux G, Pinloche S. PermutMatrix: a graphical environment to arrange gene expression profiles in optimal linear order. Bioinformatics. 2005;21:1280-1. 